\title{
Prognostic Nutritional Index Predicts Treatment Outcomes following Palliative Surgery for Colorectal Adenocarcinoma
}

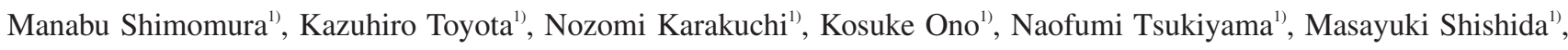 \\ Koichi Oishi ${ }^{1)}$, Kazuaki Miyamoto ${ }^{1)}$, Masahiro Ikeda ${ }^{1)}$, Seiji Sadamoto ${ }^{1)}$ and Tadateru Takahashi ${ }^{12)}$ \\ 1) Department of Surgery, National Hospital Organization Higashihiroshima Medical Center, Hiroshima, Japan \\ 2) Department of Gastroenterological and Transplant Surgery, Applied Life Sciences, \\ Institute of Biomedical \& Health Sciences, Hiroshima University, Hiroshima, Japan
}

\begin{abstract}
:
Objectives: Palliative surgeries such as stoma creation and bypass are effective for relieving symptoms related to incurable abdominal malignancies; however, these methods are controversial in patients with severe metastatic disease or poor pre-surgical health. The aim of this study was to examine the clinical significance of the prognostic nutritional index (PNI) in evaluations for palliative surgery. Methods: We retrospectively analyzed data from 37 patients who underwent palliative surgery for histologically-proven colorectal adenocarcinoma from 2009 to 2015. We investigated both risk factors for postoperative complications and prognostic factors. We used a PNI cutoff value of 40, as defined by previous studies. Results: The reason for surgery was stenosis in 18 patients, obstruction in 12, fistula in 5, and bleeding in 2 . Bypass was performed in 10 cases, ileostomy in 5, and colostomy in 22. Postoperative morbidity and mortality occurred in 9 and 2 patients, respectively. Median overall survival time was 8.9 months. Only low PNI correlated with postoperative complications at trend-level $(\mathrm{p}=0.07)$, and the 2 patients with mortality were classified as PNI-low. The presence of ascites $(\mathrm{p}=0.003)$ and PNI $(\mathrm{p}=0.02)$ were identified as independent prognostic factors. Conclusions: PNI could be used as an objective marker for deciding whether to proceed with palliative surgery, independent of the extent of metastatic disease.

Keywords:
\end{abstract}

colorectal adenocarcinoma, palliative surgery, prognostic nutritional index

J Anus Rectum Colon 2017; 1(4): 118-124

\section{Introduction}

Acute and subacute intestinal obstruction, pain, fistula, and bleeding are common occurrences among patients with incurable, advanced abdominal malignancies ${ }^{1)}$. These symptoms have a significant impact on the quality of life of patients and their families. Malignant bowel obstruction (MBO) is the most frequent of these conditions, and is estimated to occur in $10-28.4 \%$ of cases of colorectal cancer $(\mathrm{CRC})^{2)}$.

Palliative surgical procedures, such as stoma creation and bypass, are effective treatment methods to relieve these symptoms; however, these approaches are controversial in cases of severe metastatic disease or poor patient health. In cases of $\mathrm{MBO}$, recent studies have reported the efficacy of alternative treatments such as self-expandable metallic stents $(\text { SEMS })^{3)}$ or medical management with agents such as octreotide $^{1)}$. The establishment of a marker that can identify an optimal group for surgical intervention will help to minimize the risks of unnecessary surgery and allow for the application of less-invasive alternative therapies.

The prognostic nutritional index (PNI), which is calculated based on the serum albumin concentration and total lymphocyte count in the peripheral blood, was initially proposed by Onodera and colleagues to assess the nutritional and immunological status (and thus surgical risk) of patients undergoing gastrointestinal surgery ${ }^{4}$. Previous studies have reported the predictive potential of the PNI on prognosis of 
several malignancies, including $\mathrm{CRC}^{5}$. However, there have been no investigations to date of whether the PNI predicts treatment outcomes after palliative surgery for CRC.

In the present study, we investigated both risk factors for postoperative complications and prognostic factors following palliative surgery. The aim of this study was to examine the clinical significance of the PNI in evaluations for palliative surgery.

\section{Methods}

We retrospectively analyzed data from 37 patients with histologically-proven colorectal adenocarcinoma who underwent palliative surgery from 2009 to 2015 at the Higashihiroshima Medical Center. Patient characteristics and treatment outcomes were obtained from an established database, and we subsequently evaluated treatment outcomes of palliative surgery. We investigated risk factors for postoperative complications as well as prognostic factors, and determined the clinical impact of the PNI after palliative surgery. Patients who underwent curative surgery after palliative surgery or gastrojejunostomy were excluded. During the study period, there were no cases of stent placement because of issues pertaining to instruments and technical difficulties.

The following clinicopathological variables were investigated: sex (male/female), age (low/high), operative method (stoma/bypass), presence of bowel obstruction (present/absent), presence of metastatic disease (present/absent), presence of peritoneal dissemination (present/absent), extent of peritoneal dissemination (P0-P2/P3), positivity of peritoneal lavage (positive/negative), preoperative carcinoembryonic antigen (CEA) serum level (low/high), presence of primary tumor resection (yes/no), presence of preoperative chemotherapy (yes/no), Eastern Cooperative Oncology Group Performance Status (ECOG PS; 0, 1/2, 3), preoperative body mass index (low/high), albumin level (low/high), lymophocytes count (low/high), and PNI (low/high). The cutoff values for the continuous variables, with the exception of the PNI, were determined by median value. The cutoff value of the PNI was determined to be 40 , as defined by Onodera and colleagues $^{4}$.

The study was approved by the local ethics committee. Survival time was calculated from the date of palliative surgery.

\section{Surgery}

Cytology was performed if ascites was present at the time of laparotomy. If ascites was absent, peritoneal lavage was performed. We aimed to conduct bypass operations if possible, in cases where no stenotic lesions were observed in the pelvic cavity. When it was inappropriate to perform bypass surgery, we aimed to perform a colostomy if there were stenotic lesions in the distal colon. When it was inappropriate to perform colostomy, we aimed to perform a ileostomy.

\section{Definition}

The extent of peritoneal dissemination was defined according to the Japanese Classification of Colorectal Carcinoma ${ }^{6}$ : P0 (absence of dissemination), P1 (localized dissemination in peritoneum near primary tumor), P2 (localized dissemination in peritoneum distant from primary tumor), and P3 (diffuse dissemination).

Postoperative complications were reviewed for at least 30 days after surgery. The complications were graded according to the method described by Dindo and colleagues ${ }^{7}$. Complications with a grade above 2 were classified as morbid. Postoperative mortality was defined as any death that occurred within 30 days of surgery.

\section{Statistical analyses}

The differences between the two groups were analyzed using the chi-square test and the Mann-Whitney U-test. Survival curves were plotted using the Kaplan-Meier method, and univariate analyses were estimated using the log-rank test. The Cox proportional hazards model was used for multivariate analyses. In all analyses, statistical significance was set at a $p$ value less than 0.05 . All statistical analyses were performed using the IBM SPSS Statistics 20.0 software package (IBM Corp., Armonk, NY, USA).

\section{Results}

\section{Patient characteristics}

23 male and 14 female patients with a median age of 75 years (range, 48-92 years) were included in the study. The reason for surgery was stenosis in 18 cases, obstruction in 12 , fistula in 5 , and bleeding in 2. Bypass was performed in 10 cases, ileostomy in 5 cases, and colostomy in 22 cases. Prior to the present surgery, primary tumor resection had been performed in 6 cases, and chemotherapy had been administered in 7 cases. Distant metastases were present in 26 cases, and ascites was observed in 16 cases during surgery. Peritoneal cytology (including lavage) was performed in 31 cases, and cytology was positive in 7 cases. The median serum CEA level was $25.3 \mathrm{ng} / \mathrm{mL}$ (1.6-5749). Median PNI was 39.6 (range, 32.1-53.2). Patient characteristics are shown in Table 1. The median follow-up time was 7.4 months (range, 0.2-76.8).

\section{Treatment outcomes}

Morbidity was observed in 8 patients $(22.0 \%)$, while mortality occurred in 2 patients (5.4\%). Specifically, pneumonia occurred in 4 patients, intraabdominal abscess in 2 patients, ileus in 1 patient, and disseminated intravascular coagulation in 1 patient. Oral intake was enabled in 36 cases (97\%), and discharge was possible in 25 cases (68\%). Recurrent bowel obstruction within the survival time was observed in only 1 case $(2.7 \%)$. Postoperative chemotherapy was performed in 21 cases (57\%), and median overall survival time was 8.9 
Table 1. Patient characteristics $(n=37)$.

\begin{tabular}{|c|c|c|}
\hline Sex & Male/Female & $23 / 14$ \\
\hline Median age (range) & & $75(48-92)$ \\
\hline \multicolumn{3}{|l|}{ Diagnosis } \\
\hline & Colon cancer & 20 \\
\hline & Rectal cancer & 17 \\
\hline \multicolumn{3}{|l|}{ Reason for operation } \\
\hline & Stenosis & 18 \\
\hline & Obstruction & 12 \\
\hline & Fistula & 5 \\
\hline & Bleeding & 2 \\
\hline \multicolumn{3}{|l|}{ Operative methods } \\
\hline & Bypass & 10 \\
\hline & Ileostomy & 5 \\
\hline & Colostomy & 22 \\
\hline Preoperative chemotherapy & & 7 \\
\hline Primary tumor resection & & 6 \\
\hline Distant metastasis & & 26 \\
\hline Ascites & Present & 16 \\
\hline Peritoneal cytology (Including lavage) & Positive & 7 \\
\hline \multirow[t]{2}{*}{ Histological type } & Well, Mod & 34 \\
\hline & Poorly & 3 \\
\hline \multirow[t]{4}{*}{ The extent of peritoneal dissemination } & P0 & 23 \\
\hline & $\mathrm{P} 1$ & 4 \\
\hline & $\mathrm{P} 2$ & 2 \\
\hline & P3 & 9 \\
\hline Median serum CEA levels (ng/ml) (range) & & $16.2(1.6-5749)$ \\
\hline \multirow[t]{2}{*}{ ECOG Performance Status } & $0-1$ & 28 \\
\hline & $2-$ & 9 \\
\hline Median Body Mass index (range) & & $19.9(14.2-35.5)$ \\
\hline Median albumin (g/dl) (range) & & $3.4(2.3-4.4)$ \\
\hline Median lymphocytes (/ $\mu \mathrm{l})$ (range) & & $1420(430-2170)$ \\
\hline Median PNI (range) & & $41.1(31.2-53.2)$ \\
\hline
\end{tabular}

Well well differentiated adenocarcinoma, Mod moderately differentiated adenocarcinoma, Poorly poorly differentiated adenocarcinoma, CEA carcinoembryonic antigen, ECOG Eastern Cooperative Oncology Group, PNI prognostic nutritional index

months (Figure 1A).

\section{Risk factors for postoperative complications}

To investigate risk factors for postoperative complications, univariate analyses were performed for each variable. There were no significant risk factors, however, low PNI correlated with postoperative complications at trend-level $(\mathrm{p}=0.07)(\mathrm{Ta}-$ ble 2 ). The 2 patients who experienced mortality were classified as PNI-low.

\section{Prognostic factors}

To investigate prognostic factors, univariate analyses were performed for the variables, including postoperative complications. The extent of peritoneal dissemination ( $\mathrm{P} 3, p=0.04$ ), the presence of ascites $(p=0.006)$, poor ECOG PS ( 2 or more, $p=0.02$ ), and PNI (low, $p=0.02$ ) were identified as significant prognostic factors (Table 3 ). In a multivariate analysis of the variables found to be significant in the univariate analyses, the presence of ascites $(p=0.003)$ and PNI ( $p=$ 0.02 ) were identified as independent prognostic factors (Ta- ble 3).

The median survival time of patients classified as PNIlow was 5.6 months, whereas patients classified as PNI-high had a median survival time of 24.1 months ( $p=0.02$, Figure 1B). Long-term outcomes were not expected in patients classified as PNI-low.

\section{Discussion}

In the present study, we demonstrated that palliative surgeries are effective treatment methods that enable most patients to achieve oral intake within their remaining lifetimes. Moreover, we showed that the PNI predicted short- and long-term outcomes, independent of the extent of metastatic disease.

Palliative surgeries have been reported to be effective treatment approaches for the relief of malignant symptoms in the abdominal cavity; however, high morbidity and mortality have also been reported due to poor patient health presurgery. Blair and colleagues reported a treatment-related 

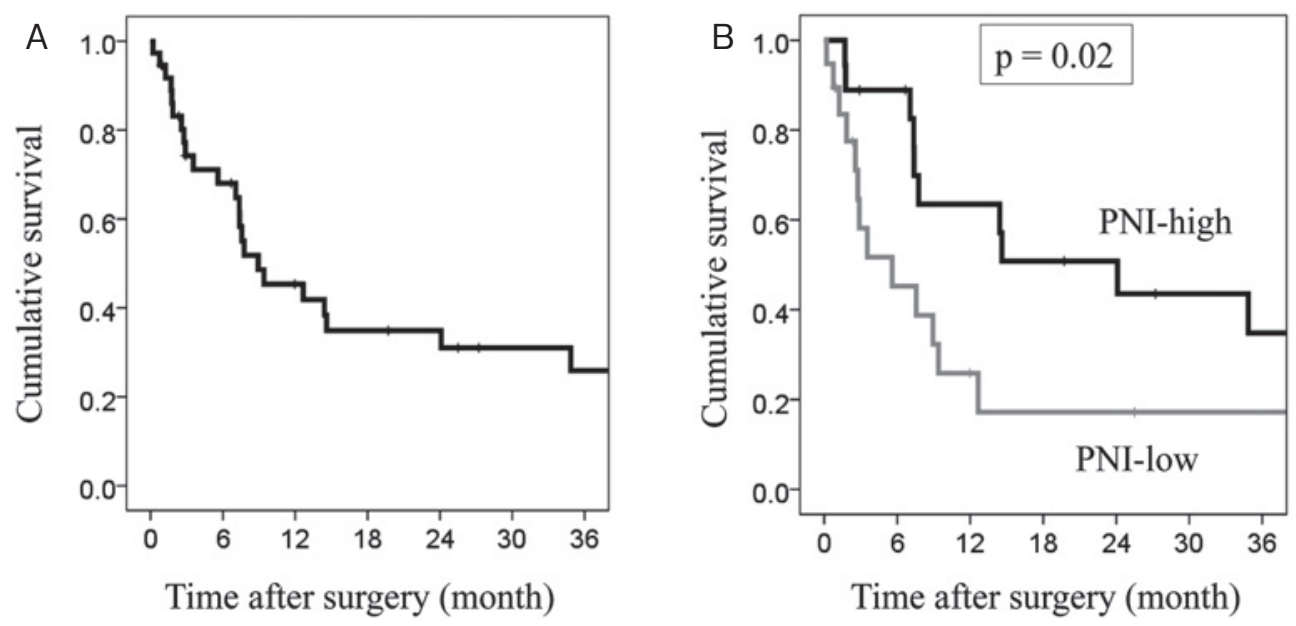

Figure 1. A: Overall survival after palliative surgery. Median overall survival time was 8.9 months.

B: Prognostic utility of the prognostic nutritional index (PNI). The median survival time of patients with low PNI was 5.6 months, whereas that of patients with high PNI was 24.1 months.

morbidity rate of $44 \%$ and a mortality rate of $15 \%$ in 63 cases after palliative surgery for gastrointestinal malignan$\operatorname{cies}^{8}$. Previous studies also reported a limited median survival time of about 4 to 7 months after surgery ${ }^{9,10)}$. The process of surgical decision-making relies upon an assessment of the likely benefits and risks to the patient of the proposed procedure $^{1)}$. Despite the important role of palliative surgery in maintaining the quality of life of patients with incurable diseases, few reports have examined the outcomes of palliative surgical procedures. A previous study examined patients who had ascites and cancer, which first presented with $\mathrm{MBO}$, and found that patients with noncolorectal primary tumors had inferior survival ${ }^{8}$. Higashi and colleagues reported that the presence of more than $100 \mathrm{~mL}$ of ascetic fluid was related to short symptom-free survival ${ }^{10)}$; however, objective criteria for palliative surgery have not been determined. Therefore, the actual indications for surgery have been dependent on the judgement of surgeons at each institution, and the establishment of selection criteria to improve treatment outcomes for palliative surgery is sorely needed. In the present study, we demonstrated that the PNI predicted short- and long-term outcomes following palliative surgery. These results indicated that the PNI could be used as an objective marker for deciding whether to proceed with palliative surgery, independent of the extent of metastatic disease.

The presence of ascites was also identified as an independent prognostic factor in the present study; therefore, careful decision-making should take this factor into consideration when relevant for a patient, along with the PNI. Ascites in patients with advanced cancer likely develops in the following manner: cancer cells produce excessive fluid, but the lymph glands in the abdomen are blocked and cannot drain away the excess fluid adequately. Additionally, the liver cannot synthesize sufficient quantities of blood proteins. Low nutritional status and a past history of heart, liver, and renal failure in the patient can also contribute to the presence of ascites. Therefore, the causes of ascites in patients with advanced cancer could be attributed to a number of etiological factors ${ }^{11}$. This suggests that a single cause of ascites does not have significant prognostic value, as multiple factors including low nutrition, cancer cachexia, and organ failure influence the prognosis.

Determinants of cancer progression and prognosis are multifactorial, and the important role of cancer-associated malnutrition has been increasingly recognized ${ }^{12}$. Over the past decades, it has become well-accepted that malnutrition is associated with poorer response to therapy, greater impairment of quality of life, increased risk of chemotherapyinduced toxicity, and worse outcomes ${ }^{13-15}$. Assessment of the systemic immunonutritional status has been refined by the introduction of the PNI, and many studies have reported the significant prognostic role of this variable for several malignancies. The PNI can be calculated from the serum albumin concentration and lymphocyte count, which are standard parameters assessed in a clinical setting. Cancer progression worsens nutritional status and can cause subsequent hypoalbuminemia. Poor nutritional status also causes lymphopenia, which is associated with a pre-existing state of immunosuppression, suggesting that the patient has an inadequate antitumor immunological reaction ${ }^{16}$. That indicated that cancer progression was correlated to the decline in the PNI. The present study provides the first description of the clinical significance of the PNI following palliative surgery. The significance of the other nutritional markers, such as the modified Glasgow prognostic score ${ }^{177}$, should be evaluated in future studies. In addition, the question of whether preoperative nutritional therapy can lead to improved treatment outcomes - including survival—after palliative surgery should also be investigated.

Recent studies have reported the efficacy of other palliative interventions, such as SEMS, for the treatment of malignant colonic obstruction ${ }^{3)}$. It has been reported that the 
Table 2. Risk Factors for Postoperative Complications.

\begin{tabular}{|c|c|c|c|c|}
\hline & & \multicolumn{2}{|c|}{ Postoperative complication } & \multirow[b]{2}{*}{ P-value } \\
\hline & & no & yes & \\
\hline \multirow[t]{2}{*}{ Sex } & Male & 16 & 7 & 0.27 \\
\hline & Female & 12 & 2 & \\
\hline \multirow[t]{2}{*}{ Age } & low & 14 & 3 & 0.38 \\
\hline & high & 14 & 6 & \\
\hline \multirow[t]{3}{*}{ Operative method } & ileostomy & 3 & 2 & 0.67 \\
\hline & colostomy & 17 & 5 & \\
\hline & bypass & 8 & 2 & \\
\hline \multirow[t]{2}{*}{ Bowel obstruction } & absent & 18 & 7 & 0.45 \\
\hline & present & 10 & 2 & \\
\hline \multirow[t]{2}{*}{ Distant metastasis } & absent & 9 & 2 & 0.57 \\
\hline & present & 19 & 7 & \\
\hline \multirow[t]{2}{*}{ Peritoneal dissemination } & absent & 16 & 6 & 0.61 \\
\hline & present & 12 & 3 & \\
\hline \multirow[t]{2}{*}{ The extent of peritoneal dissemination } & $\mathrm{P} 0-2$ & 22 & 6 & 0.47 \\
\hline & P3 & 6 & 3 & \\
\hline \multirow[t]{2}{*}{ Ascites } & absent & 16 & 5 & 0.93 \\
\hline & present & 12 & 4 & \\
\hline \multirow[t]{2}{*}{ Peritoneal cytology } & negative & 18 & 6 & 0.85 \\
\hline & positive & 5 & 2 & \\
\hline \multirow[t]{2}{*}{ Serum CEA levels } & low & 13 & 3 & 0.49 \\
\hline & high & 15 & 6 & \\
\hline \multirow[t]{2}{*}{ Resection of the primary tumor } & no & 23 & 8 & 0.63 \\
\hline & yes & 5 & 1 & \\
\hline \multirow[t]{2}{*}{ Previous treatment } & no & 21 & 9 & 0.10 \\
\hline & yes & 7 & 0 & \\
\hline \multirow[t]{2}{*}{ Infectious disease } & absent & 19 & 6 & 0.95 \\
\hline & present & 9 & 3 & \\
\hline \multirow[t]{2}{*}{ ECOG Performance Status } & $0-1$ & 23 & 5 & 0.11 \\
\hline & $2-$ & 5 & 4 & \\
\hline \multirow[t]{2}{*}{ Body mass index } & low & 14 & 4 & 0.77 \\
\hline & high & 14 & 5 & \\
\hline \multirow[t]{2}{*}{ Albumin } & low & 17 & 6 & 0.75 \\
\hline & high & 11 & 3 & \\
\hline \multirow[t]{2}{*}{ lymphocytes } & low & 13 & 7 & 0.10 \\
\hline & high & 15 & 2 & \\
\hline \multirow[t]{2}{*}{ PNI } & low & 12 & 7 & 0.07 \\
\hline & high & 16 & 2 & \\
\hline
\end{tabular}

$\overline{\text { CRC colorectal cancer, } C E A \text { carcinoembryonic antigen, ECOG Eastern Cooperative Oncology Group, PNI }}$ prognostic nutritional index

technical success rate of this procedure ranges from 88$100 \%$, whereas the migration rate is $11 \%$ and the perforation rate is $4.5 \%$ in the palliative setting. Although the initial clinical relief of obstruction was higher after palliative surgery $(100 \%)$ compared with stent placement $(93 \%)$, a significantly lower 30-day mortality rate and shorter duration of hospitalization were reported with SEMS ${ }^{18}$. European Society of Gastrointestinal Endoscopy (ESGE) guidelines recommend SEMS placement as the preferred treatment for palliation in selected patients with malignant colonic obstruction $^{33}$. Despite the high success rate of SEMS, however, long-term patency is not guaranteed; the median patency time has been reported to be 106 days (68-288 days) and the patency rate was found to be $80 \%$ (53-90\%) until death $^{19)}$. In addition, chemotherapy with antiangiogenic agents, which is usually used for gastric cancer and CRC, has been found to be a risk factor for perforation after SEMS insertion ${ }^{20}$. In the present study, the median survival time of patients classified as PNI-high was 24.1 months, whereas that of patients classified as PNI-low was 5.6 months. These findings suggest that patients with high PNI and a life expectancy of more than 1 year should undergo palliative surgery even in cases in which SEMS insertion is possible.

For patients with bleeding, pain, or fistula, medical palliation is not expected to be effective. Only surgical palliation could improve the quality of life for such patients, despite the high morbidity and mortality rates. We believe, there- 
Table 3. Prognostic Factors.

\begin{tabular}{|c|c|c|c|c|c|c|c|}
\hline & & \multirow{2}{*}{$\mathrm{n}$} & \multirow{2}{*}{$\begin{array}{c}\text { median OS } \\
\text { (month) }\end{array}$} & \multirow{2}{*}{$\frac{\text { univariate }}{\mathrm{P} \text {-value }}$} & \multicolumn{3}{|c|}{ multivariate } \\
\hline & & & & & P-value & Odds ratio & $95 \%$ Confidence interval \\
\hline \multirow[t]{2}{*}{ Sex } & Male & 23 & 14.6 & 0.11 & & & \\
\hline & Female & 14 & 7.8 & & & & \\
\hline \multirow[t]{2}{*}{ Age } & low & 17 & 12.3 & 0.62 & & & \\
\hline & high & 20 & 7.8 & & & & \\
\hline \multirow[t]{2}{*}{ Operative method } & stoma & 31 & 12.3 & 0.65 & & & \\
\hline & bypass & 10 & 7.6 & & & & \\
\hline \multirow[t]{2}{*}{ Bowel obstruction } & absent & 25 & 8.9 & 0.37 & & & \\
\hline & present & 12 & 9.4 & & & & \\
\hline \multirow[t]{2}{*}{ Distant metastasis } & absent & 11 & 14.4 & 0.50 & & & \\
\hline & present & 26 & 7.6 & & & & \\
\hline \multirow[t]{2}{*}{ Peritoneal dissemination } & absent & 22 & 14.4 & 0.09 & & & \\
\hline & present & 15 & 7.4 & & & & \\
\hline \multirow[t]{2}{*}{ The extent of peritoneal dissemination } & $\mathrm{P} 0-2$ & 28 & 12.7 & 0.04 & 0.94 & 1.05 & $0.34-3.23$ \\
\hline & P3 & 9 & 7.4 & & & & \\
\hline \multirow[t]{2}{*}{ Ascites } & absent & 21 & 14.4 & 0.006 & 0.003 & 5.68 & $1.83-17.5$ \\
\hline & present & 16 & 3.6 & & & & \\
\hline \multirow[t]{2}{*}{ Peritoneal cytology } & negative & 26 & 14.4 & 0.25 & & & \\
\hline & positive & 9 & 15.2 & & & & \\
\hline \multirow[t]{2}{*}{ Serum CEA levels } & low & 16 & 7.6 & 0.28 & & & \\
\hline & high & 21 & 14.6 & & & & \\
\hline \multirow[t]{2}{*}{ Resection of the primary tumor } & no & 31 & 7.6 & 0.17 & & & \\
\hline & yes & 6 & 24.1 & & & & \\
\hline \multirow[t]{2}{*}{ Previous treatment } & no & 30 & 9.4 & 0.45 & & & \\
\hline & yes & 7 & 7.4 & & & & \\
\hline \multirow[t]{2}{*}{ Infectious disease } & absent & 25 & 8.9 & 0.87 & & & \\
\hline & present & 12 & 12.7 & & & & \\
\hline \multirow[t]{2}{*}{ Postoperative complication } & absent & 28 & 9.4 & 0.36 & & & \\
\hline & present & 9 & 5.6 & & & & \\
\hline \multirow[t]{2}{*}{ ECOG Performance status } & $0-1$ & 28 & 12.7 & 0.02 & 0.25 & 2.15 & $0.58-7.87$ \\
\hline & $2-$ & 9 & 2.9 & & & & \\
\hline \multirow[t]{2}{*}{ PNI } & low & 19 & 5.6 & 0.02 & 0.02 & 3.75 & $1.23-11.36$ \\
\hline & high & 18 & 24.1 & & & & \\
\hline
\end{tabular}

$\overline{O S \text { overall survival, } C R C \text { colorectal cancer, Well well differentiated adenocarcinoma, Mod moderately differentiated adenocarcinoma, Poorly poorly differentiated }}$ adenocarcinoma, CEA carcinoembryonic antigen, ECOG Eastern Cooperative Oncology Group, PNI prognostic nutritional index

fore, that the indications for surgical palliation should not be determined only by the PNI in such cases.

The limitations of the present study include the fact that it is a retrospective study with a relatively small sample size.

In conclusion, the preoperative PNI predicted short- and long-term outcomes following palliative surgery. The PNI could be used as an objective marker for deciding whether to proceed with palliative surgery, independent of the extent of metastatic disease.

\section{Conflicts of Interest}

There are no conflicts of interest.

\section{References}

1. Ferguson HJ, Ferguson CI, Speakman J, et al. Management of intestinal obstruction in advanced malignancy. Ann Med Surg. 2015 Aug; 4: 264-70.

2. Ripamonti C, De Conno F, Ventafridda V, et al. Management of bowel obstruction in advanced and terminal cancer patients. Ann Oncol. 1993 Jan; 4: 15-21.

3. van Hooft JE, van Halsema EE, Vanbiervliet G, et al. Selfexpandable metal stents for obstructing colonic and extracolonic cancer: European Society of Gastrointestinal Endoscopy (ESGE) Clinical Guideline. Endoscopy. 2014 Nov; 46: 990-1053.

4. Onodera T, Goseki N, Kosaki G. Prognostic nutritional index in gastrointestinal surgery of malnutrished cancer patients. Nihon Geka Gakkai Zasshi. 1984 Sep; 85: 1001-5.

5. Mohri Y, Inoue Y, Tanaka K, et al. Prognostic nutritional index predicts postoperative outcome in colorectal cancer. World J Surg. 2013 Nov; 37: 2688-92.

6. Japanese Society for Cancer of the Colon and Rectum. General Rules for Clinical and Pathological Studies on Cancer of the Colon, Rectum and Anus. 8th ed. Tokyo: Kanehara Shuppan, 2013.

7. Dindo D, Demartines N, Clavien PA. Classification of surgical complications: a new proposal with evaluation in a cohort of 6336 patients and results of a survey. Ann Surg. 2004 Aug; 240: 20513.

8. Blair SL, Chu DZ, Schwarz RE. Outcome of palliative operations 
for malignant bowel obstruction in patients with peritoneal carcinomatosis from nongynecological cancer. Ann Surg Oncol. 2001 Sep; 8: 632-7.

9. Soriano A, Davis MP. Malignant bowel obstruction: individualized treatment near the end of life. Cleve Clin J Med. 2011 Mar; 78: 197-206.

10. Higashi H, Shida H, Ban K, et al. Factors affecting successful palliative surgery for malignant bowel obstruction due to peritoneal dissemination from colorectal cancer. Jpn J Clin Oncol. 2003 Jul; 33: 357-9.

11. Stukan M. Drainage of malignant ascites: patient selection and perspectives. Cancer Manag Res. 2017 Apr; 9: 115-30.

12. McMillan DC. Systemic inflammation, nutritional status and survival in patients with cancer. Curr Opin Clin Nutr Metab Care. 2009 May; 12: 223-6.

13. Liu X, Qiu H, Liu J, et al. A Novel Prognostic Score, Based on Preoperative Nutritional Status, Predicts Outcomes of Patients after Curative Resection for Gastric Cancer. J Canc. 2016 Oct; 7: 214856.

14. Sala A, Rossi E, Antillon F, et al. Nutritional status at diagnosis is related to clinical outcomes in children and adolescents with cancer: a perspective from Central America. Eur. J Canc. 2012 Jan; 48: $243-52$.
15. von Meyenfeldt M. Cancer-associated malnutrition: an introduction. Eur J Oncol Nurs. 2005; 9 Suppl 2: S35-8.

16. Shibutani M, Maeda $K$, Nagahara $H$, et al. The prognostic significance of the postoperative prognostic nutritional index in patients with colorectal cancer. BMC cancer $2015 \mathrm{Jul}$; 15: 521.

17. McMillan DC, Crozier JE, Canna K, et al. Evaluation of an inflammation-based prognostic score (GPS) in patients undergoing resection for colon and rectal cancer. Int J Colorectal Dis. 2007 Aug; 22: 881-6.

18. Liang TW, Sun Y, Wei YC, et al. Palliative treatment of malignant colorectal obstruction caused by advanced malignancy: a selfexpanding metallic stent or surgery? A system review and metaanalysis. Surg Today. 2014 Jan; 44: 22-3.

19. Watt AM, Faragher IG, Griffin TT, et al. Self-expanding metallic stents for relieving malignant colorectal obstruction: a systematic review. Ann Surg. 2007 Jul; 246: 24-30.

20. van Halsema EE, van Hooft JE, et al. Perforation in colorectal stenting: a meta-analysis and a search for risk factors. Gastrointest Endosc. 2014 Jun; 79: 970-82 e977; quiz 983 e972, 983 e975.

Journal of the Anus, Rectum and Colon is an Open Access article distributed under the Creative Commons Attribution-NonCommercial-NoDerivatives 4.0 International License. To view the details of this license, please visit (https://creativecommons.org/licenses/by-nc-nd/4.0/). 